\title{
The evolutionary nature of the cancer immunotherapy revolution
}

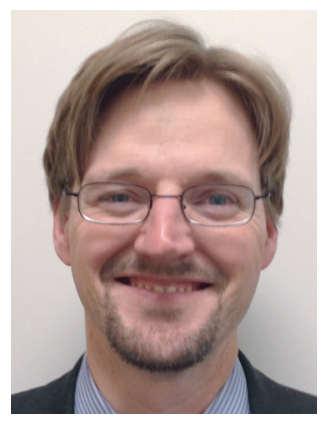

Ryan J Sullivan ${ }^{*}$

\author{
"Cancer immunotherapy, a field that had too few \\ breakthroughs in the 20th century to inspire the masses, \\ is the hottest topic in oncology."
}

First draft submitted 15 May 2017; Accepted for pub+lication 17 May 2017; Published online: 22 August 2017

Cancer immunotherapy, a field that had too few breakthroughs in the 20th century to inspire the masses, is the hottest topic in oncology. From dominating abstracts and presentations at major scientific meetings to a proliferation of articles in the most prestigious journals and even direct-toconsumer advertising, the totality of the shift from full scale denying and denouncing the utility of immunotherapy by the medical oncology community to full-on acceptance has been astonishing. This shift is entirely related to major advances into the understanding of how the immune system works, interacts with tumors and vice versa, and is regulated. Only after this foundation work was established did the clinical development proceed and succeed.

The origins of modern cancer immunotherapy are traced to William Coley, who developed a bacterial toxin designed to stimulate an immune activation that somewhat magically would trigger a tumor-specific response [1]. This approach was never proven to be effective in randomized studies, but yet served as an important proof of principle. The next breakthrough was discovering that $\mathrm{T}$ cells were key effectors of a tumor-specific immune response and that a T-cell growth factor, otherwise known as interleukin 2 (IL-2), could further propagate these responses [2,3]. In fact, high-dose IL-2 was FDA-approved in the 1990s for both metastatic renal cell carcinoma and metastatic melanoma; two diseases where traditional therapies such as cytotoxic chemotherapy did not work particularly well $[4,5]$. During this same era, a number of approaches to prime and direct tumor antigen-specific immunity were designed through a series of vaccines given with a variety of adjuvants. These efforts could almost always be shown to trigger immune responses, typically through ex vivo stimulation assays, in some patients but almost never lead to clinical benefit [6].

Through two major breakthroughs in the late 20th century, the way we approach the treatment of patients with solid tumors with immune directed therapy has changed forever. First, the pioneering work of the Surgical Branch at the National Cancer Center (MD, USA), under the leadership of Steven Rosenberg, established standard operating procedures to isolate tumor infiltrating lymphocytes, activate and expand these ex vivo, and infuse following conditioning regimens [7,8]. These efforts have either directly or indirectly inspired the development of $\mathrm{T}$ cell products with engineered T-cell receptors (TCRs) or chimeric antigen receptors (CARs), which activate specific antigens expressed on tumor cells and direct a potent and specific antigen-specific T cell mediated immunity. Second, the

'Massachusetts General Hospital \& Harvard Medical School, Boston, MA, USA

*Author for correspondence: Fax: 617724 3166; Tel.: 617724 4000; rsullivan7@partners.org
“Through two major breakthroughs in the late 20th century, the way we approach the treatment of patients with solid tumors with immune directed therapy has changed forever,"
Future 
"In this issue of Future Oncology entitled 'Cancer immunotherapies and the potential for combination', cancer immunotherapy is the topic and the development of potential combinatorial regimens the focus." identification of immune regulatory elements and agents to disrupt these so-called immune checkpoints has changed the narrative about immunotherapy from being a niche type of treatment for a small number of not particularly common diseases to being the type of therapy that every pharmaceutical and small biotech company must account for, leading to the development of entire immuno-oncology (IO) programs because responses and improved outcomes have been seen in subsets of patients with common malignancies, including lung, breast and colorectal cancer $[9,10]$.

The driving factor for the $\mathrm{IO}$ revolution focuses around the finding that $\mathrm{B} 7$ homolog $1(\mathrm{~B} 7-\mathrm{H} 1$; PD-L1), which may be expressed in tumors in response to $\mathrm{T}$ cell infiltration, serves as a ligand to the Programmed Death 1 receptor (PD-1), which is expressed in activated $T$ effector cells [9]. When PD-1 and PD-L1 interact, the activated T cells are blocked entry into the tumor. Inhibition of this interaction leads to effective infiltration of activated $\mathrm{T}$ cells and ultimately tumor destruction [11]. Remarkably, monoclonal antibodies against PD-1 (pembrolizumab, nivolumab) and PD-L1 (atezolizumab, avelumab, durvalumab) have received regulatory approval in a number of diseases, including melanoma, non-small-cell lung cancer (NSCLC), urothelial bladder cancer (UBC), head and neck squamous cell carcinoma (HNSCC), classical Hodgkin lymphoma and Merkel cell carcinoma, and have shown activity in many others [12-21]. Building upon this work, data with combination therapy with anti-PD-1/ PD-L1 agents with anticytotoxic T lymphocyte associated antigen 4 (CTLA4) agents, such as ipilimumab, which was FDA-approved as a single agent in metastatic melanoma in 2011, and tremelimumab, is associated with higher response rates in a number of diseases [22-28].

However, despite the promise of immunotherapy in 2017, a number of limitations prevent the widespread use of these agents for the majority of cancer patients. First, the stark reality is that the majority of patients will not respond to these treatments and cures, to date represented by the rarity of rates of durable responses lasting at least a few years. Second, few biomarkers to help prospectively select patients for these therapies exist. Third, very little is known about the mechanisms driving primary and acquired resistance to therapy. Fourth, the toxicity of immunotherapy is not trivial and provides a robust challenge, particularly in the development of more effective combinations. Finally, the cost of many of these agents is astronomical and, particularly if at some point these agents become standard for most cancer indications, unsustainable. To say there is more work to be done is a gross understatement.

In this issue of Future Oncology entitled "Cancer immunotherapies and the potential for combination", cancer immunotherapy is the topic and the development of potential combinatorial regimens the focus. With nearly limitless potential combinations and hundreds to thousands of clinical trials ongoing or being planned, it is safe to say this is a broad topic. The papers contained within are thus diverse, both with regard to disease (gastroesophageal adenocarcinoma, colorectal cancer, NSCLC, urothelial cancer, virally-mediated cancers) and types of immunotherapy (peptide vaccines, CAR T cells, anti-PD-1/PD-L1, antiangiogenic therapy, local therapies, oncogene targeted therapy, chemotherapy, radiation therapy), but by no means representative of the entirety of the field of combination immunotherapy. Rather, what is presented herein is a primer of where the field currently is and where it is going. The future is indeed bright and the challenges are profound but also surmountable.

\section{Financial \& competing interests disclosure}

The authors have no relevant affiliations or financial involvement with any organization or entity with a financial interest in or financial conflict with the subject matter or materials discussed in the manuscript. This includes employment, consultancies, honoraria, stock ownership or options, expert testimony, grants or patents received or pending, or royalties.

No writing assistance was utilized in the production of this manuscript.

\section{Reference}

1 Coley WB. The treatment of inoperable sarcoma by bacterial toxins (the mixed toxins of the Streptococcus erysipelas and the Bacillus prodigiosus). Proc. R. Soc. Med. 3(Surg Sect), 1-48 (1910).
2 Rosenberg SA, Eberlein TJ, Grimm EA, Lotze MT, Mazumder A, Rosenstein M. Development of long-term cell lines and lymphoid clones reactive against murine and human tumors: a new approach to the adoptive immunotherapy of cancer. Surgery 92(2), 328-336 (1982).
3 Atkins MB, Lotze MT, Dutcher JP et al. High-dose recombinant interleukin 2 therapy for patients with metastatic melanoma: analysis of 270 patients treated between 1985 and 1993. J. Clin. Oncol. 17(7), 2105-2116 (1999). 
4 Fyfe G, Fisher RI, Rosenberg SA, Sznol M, Parkinson DR, Louie AC. Results of treatment of 255 patients with metastatic renal cell carcinoma who received high-dose recombinant interleukin-2 therapy. J. Clin. Oncol. 13(3), 688-696 (1995).

5 Pass HA, Schwarz SL, Wunderlich JR, Rosenberg SA. Immunization of patients with melanoma peptide vaccines: immunologic assessment using the ELISPOT assay. Cancer J. Sci. Am. 4(5), 316-323 (1998).

6 Rosenberg SA, Lotze MT, Muul LM et al. Observations on the systemic administration of autologous lymphokine-activated killer cells and recombinant interleukin- 2 to patients with metastatic cancer. New Engl. J. Med. 313(23), 1485-1492 (1985).

7 Rosenberg SA, Yang JC, Sherry RM et al. Durable complete responses in heavily pretreated patients with metastatic melanoma using T-cell transfer immunotherapy. Clin. Cancer Res. 17(13), 4550-4557 (2011).

8 Freeman GJ, Long AJ, Iwai Y et al. Engagement of the PD-1 immunoinhibitory receptor by a novel B7 family member leads to negative regulation of lymphocyte activation. J. Exp. Med. 192(7), 1027-1034 (2000).

9 Walunas TL, Lenschow DJ, Bakker CY et al. CTLA-4 can function as a negative regulator of $\mathrm{T}$ cell activation. Immunity 1(5), 405-413 (1994).

10 Topalian SL, Hodi FS, Brahmer JR et al. Safety, Activity, and immune correlates of anti-PD-1 antibody in cancer. New Engl. J. Med. doi:10.1056/NEJMoa1200690 (2012).

11 Robert C, Schachter J, Long GV et al. Pembrolizumab versus Ipilimumab in advanced melanoma. N. Engl. J. Med. 372(26), 2521-2532 (2015).

12 Robert C, Long GV, Brady B et al. Nivolumab in previously untreated melanoma without BRAF mutation. N. Engl. J. Med. doi:10.1056/NEJMoa1412082 (2014).

13 Herbst RS, Soria JC, Kowanetz M et al. Predictive correlates of response to the anti-PD-L1 antibody MPDL3280A in cancer patients. Nature 515(7528), 563-567 (2014).

14 Powles T, Eder JP, Fine GD et al. MPDL3280A (anti-PD-L1) treatment leads to clinical activity in metastatic bladder cancer. Nature 515(7528), 558-562 (2014).

15 Gettinger S, Rizvi NA, Chow LQ et al. Nivolumab monotherapy for first-line treatment of advanced non-small-cell lung cancer. J. Clin. Oncol. 34(25), 2980-2987 (2016).

16 Reck M, Rodriguez-Abreu D, Robinson AG et al. Pembrolizumab versus chemotherapy for PD-L1-positive non-small-cell lung cancer. N. Engl. J. Med. 375(19), 1823-1833 (2016).

17 Ansell SM, Lesokhin AM, Borrello I et al. PD-1 blockade with nivolumab in relapsed or refractory Hodgkin's lymphoma. N. Engl. J. Med. 372(4), 311-319 (2015).

18 Kaufman HL, Russell J, Hamid O et al. Avelumab in patients with chemotherapyrefractory metastatic Merkel cell carcinoma: a multicentre, single-group, open-label, Phase 2 trial. Lancet Oncol. 17(10), 1374-1385 (2016).

19 Bauml J, Seiwert TY, Pfister DG et al. Pembrolizumab for platinum- and cetuximabrefractory head and neck cancer: results from a single-arm, Phase II Study. J. Clin. Oncol. 35(14), 1542-1549 (2017).

20 Massard C, Gordon MS, Sharma S et al. Safety and efficacy of durvalumab (MEDI4736), an anti-programmed cell death ligand-1 immune checkpoint inhibitor, in patients with advanced urothelial bladder cancer. J. Clin. Oncol. 34(26), 3119-3125 (2016).

21 Antonia S, Goldberg SB, Balmanoukian A et al. Safety and antitumour activity of durvalumab plus tremelimumab in non-small cell lung cancer: a multicentre, Phase $1 \mathrm{~b}$ study. Lancet Oncol. 17(3), 299-308 (2016).

22 Mok T, Schmid P, Aren O et al. 192TiP: NEPTUNE: A global, Phase 3 study of durvalumab (MEDI4736) plus tremelimumab combination therapy versus standard of care (SoC) platinum-based chemotherapy in the first-line treatment of patients (pts) with advanced or metastatic NSCLC. J. Thorac. Oncol. 11(4 Suppl), S140-141 (2016).

23 Peters S, Antonia S, Goldberg SB et al. 191TiP: MYSTIC: a global, Phase 3 study of durvalumab (MEDI4736) plus tremelimumab combination therapy or durvalumab monotherapy versus platinum-based chemotherapy (CT) in the first-line treatment of patients (pts) with advanced stage IV NSCLC. J. Thorac. Oncol. 11(4 Suppl), S139-140 (2016).

24 Planchard D, Yokoi T, Mccleod MJ et al. A Phase III study of durvalumab (MEDI4736) with or without tremelimumab for previously treated patients with advanced NSCLC: rationale and protocol design of the ARCTIC study. Clin. Lung Cancer 17(3), 232-236 e231 (2016).

25 Larkin J, Chiarion-Sileni V, Gonzalez R et al. Combined nivolumab and ipilimumab or monotherapy in untreated melanoma. N. Engl. J. Med. 373(1), 23-34 (2015).

26 Postow MA, Chesney J, Pavlick AC et al. Nivolumab and ipilimumab versus ipilimumab in untreated melanoma. N. Engl. J. Med. 372(21), 2006-2017 (2015).

27 Hellmann MD, Rizvi NA, Goldman JW et al. Nivolumab plus ipilimumab as first-line treatment for advanced non-small-cell lung cancer (CheckMate 012): results of an open-label, phase 1, multicohort study. Lancet Oncol. 18(1), 31-41 (2017). 